\title{
(6) OPEN ACCESS \\ Suffering in silence: a qualitative study of second victims of adverse events
}

\author{
Susanne Ullström, Magna Andreen Sachs, Johan Hansson, \\ John Øvretveit, Mats Brommels
}

Department of Learning, Informatics, Management and Ethics (LIME), Medical Management Centre (MMC), Karolinska Institutet, Stockholm, Sweden

\section{Correspondence to}

Susanne Ullström, Department of Learning, Informatics, Management and Ethics (LIME), Medical Management Centre (MMC), Karolinska Institutet, Tomtebodavägen 18A, Stockholm 171 77, Sweden; Susanne.Ullstrom@ki.se

Received 26 March 2013 Revised 16 September 2013 Accepted 23 October 2013 Published Online First 15 November 2013

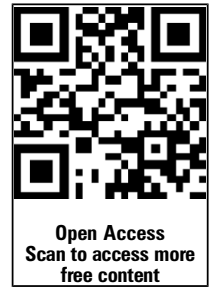

CrossMark

To cite: Ullström $\mathrm{S}$, Andreen Sachs M, Hansson J, et al. BMJ Qual Saf 2014;23: 325-331.
ABSTRACT

Introduction The term 'second victim' refers to the healthcare professional who experiences emotional distress following an adverse event. This distress has been shown to be similar to that of the patient-the 'first victim'. The aim of this study was to investigate how healthcare professionals are affected by their involvement in adverse events with emphasis on the organisational support they need and how well the organisation meets those needs.

Methods 21 healthcare professionals at a Swedish university hospital who each had experienced an adverse event were interviewed. Data from semi-structured interviews were analysed by qualitative content analysis using QSR NVivo software for coding and categorisation.

Results Our findings confirm earlier studies showing that emotional distress, often longlasting, follows from adverse events. In addition, we report that the impact on the healthcare professional was related to the organisation's response to the event. Most informants lacked organisational support or they received support that was unstructured and unsystematic. Further, the formal investigation seldom provided adequate and timely feedback to those involved. The insufficient support and lack of feedback made it more difficult to emotionally process the event and reach closure.

Discussion This article addresses the gap between the second victim's need for organisational support and the organisational support provided. It also highlights the need for more transparency in the investigation of adverse events. Future research should address how advanced support structures can meet these needs and provide learning opportunities for the organisation. These issues are central for all hospital managers and policy makers who wish to prevent and manage adverse events and to promote a positive safety culture.

\section{INTRODUCTION}

Most healthcare professionals choose their profession because they wish to improve the lives of others. When a patient is accidentally harmed in the care process, this can be a traumatic experience not only for the patient but also for the staff involved. ${ }^{12}$

Nationwide studies show that 2.9$16.6 \%$ of patients in acute care hospitals experience adverse events. ${ }^{3}$ A recent Swedish study showed that in a single year preventable adverse events may have contributed to 3000 patient fatalities and to permanent disability for 10000 patients. ${ }^{4}$ These numbers indicate that numerous healthcare professionals are likely to be involved in an adverse event at some point in their professional careers.

In recent decades, studies have reported on the emotional distress healthcare professionals experience following adverse events, ${ }^{5-15}$ insufficient organisational support $^{5} \quad 8 \quad 10-12 \quad 14$ and insufficient support from colleagues. ${ }^{6} 791315$ In the year 2000, Wu introduced the term 'second victim' to describe how healthcare professionals may be traumatised by such events in a similar way as the patient-the 'first victim'. ${ }^{16}$ Commonly reported reactions among professionals are fear, guilt, shame, self-doubt, anger and disappointment. ${ }^{1}{ }^{2}{ }^{6-14}$ In a survey of 3171 physicians in the USA and Canada, physicians reported increased anxiety about future errors, loss of self-confidence, difficulty sleeping and reduced job satisfaction following medical errors. ${ }^{10}$ Many professionals fear disciplinary action and loss of professional reputation. ${ }^{8}$

Research has shown that the impact of adverse events on the healthcare 
professional can be long-lasting, ${ }^{8} 912 \quad 13 \quad 17$ and in some instances the individual never fully recovers. ${ }^{13} \mathrm{It}$ has been suggested that the emotional reactions may be similar to those found in post-traumatic stress disorder. ${ }^{9}$ Studies report healthcare professionals consider changing career as a direct consequence of an adverse event. ${ }^{11} 13$ Others have reported a decrease in quality of life and risk of burnout, ${ }^{17}$ an increase in the use of alcohol and drugs, ${ }^{13}$ suicidal thoughts ${ }^{18}$ and even suicide. ${ }^{19}$ Scott $e t$ al conclude that regardless of gender, professional type or years in the profession, the adverse event was "a life-altering experience that left a permanent imprint on the individual" (p326). ${ }^{11}$

The way in which the organisation and managers, as well as the healthcare professional's colleagues, respond to adverse events seems to mediate the emotional impact on the healthcare professional. Adequate support can reduce the distress, while negative attitudes and lack of support can add to the emotional burden. ${ }^{10-12} 20$ In the latter instance, the organisation may end up in a vicious circle in which emotional distress and insufficient support lead to suboptimal patient care and an increased risk of future adverse events. ${ }^{117}$ Studies also report on the lack of open disclosure about adverse events in the work setting. ${ }^{5} 615$

Our overview of the literature indicates that there is scant research on the specific support that second victims need from their organisation and the extent to which the organisation meets those needs.

The aim of this study was to investigate how healthcare professionals at a Swedish university hospital were affected by their involvement in adverse events, with emphasis on the organisational support they needed and the organisational support they received.

\section{METHODS}

\section{Study setting}

The research group, with organisational assistance from the hospital's chief nursing officer and chief medical officer, conducted an interview study with employees, each of whom had been involved in an avoidable serious adverse event that had been reported to the National Board of Health and Welfare (NBHW). According to Swedish regulations, all avoidable, serious adverse events where the patient is harmed-or is at great risk of being harmed-should be reported to the NBHW, a government agency under the Ministry of Health (Management System for Systematic Quality Work (SOSFS) regulation $2005: 28$ ). It is the responsibility of every department manager to report serious adverse events, fulfilling the criteria cited above, to an assigned hospital official (often the chief medical officer), who assesses whether the event should be reported to the NBHW. If the decision is to file a report, the hospital must investigate the event using event analysis. The government agency, after further investigations, decides on whether organisational changes or interventions are required.

\section{The interview guide}

We used a semi-structured interview guide originally developed by Scott $e t$ al for their study of healthcare professionals' reactions following adverse events. ${ }^{11}$ This guide was chosen because the questions were relevant and suitable for the purpose of our study and it would also indicate whether 'second victim' responses in Sweden were similar or different to those found in the USA. We translated the guide into Swedish, with some small modifications. First, we divided the original question concerning the impact of the event into subquestions on individual well-being, the work situation and relationships with colleagues. We also added a specific question on whether organisational support was provided. The guide had 30 questions in its final from.

\section{Data gathering procedures}

The chief nursing officer prepared a list of 133 people who had each experienced a serious adverse event at the hospital in 2009 or 2010. In August 2011, we sent a letter to these people, signed by the chief nursing officer and the chief medical officer, inviting them to participate in individual interviews and to respond by telephone or email if they were interested. We guaranteed the anonymity of all participants and their hospital departments.

Of the 29 people who responded to the letter, 21 agreed to be interviewed. All interviews were conducted between September and December 2011. Of the authors, SU conducted 19 interviews, and MAS and $\mathrm{J} \varnothing$ conducted one interview each. Each interview lasted between 60 and $90 \mathrm{~min}$. We asked the informants for permission to digitally record the interviews; all except two agreed. One other interview was not recorded, making a total of three interviews in which the researcher took only handwritten notes. The 18 recorded interviews were transcribed verbatim and we verified their reliability by re-checking half of them against the recordings.

\section{The characteristics of the informants}

The occupational distribution of the 21 informants was as follows: physicians $(n=10)$, registered nurses $(n=9)$ and allied healthcare professionals $(n=2)$. Sixteen of the participants were women and five were men. The length of time in practice ranged from 5 to 30 years. The largest group $(n=9)$ had worked for 21-30 years, the second largest group $(n=7)$ for 1120 years, and the smallest group $(n=5)$ for $5-10$ years. Eighteen of the 21 informants were still employed by the hospital at the time of the interviews.

\section{Data analysis}

We used qualitative content analysis and a systematic classification process ${ }^{21} 22$ to analyse the transcribed interviews. Two authors (SU and MAS) read all transcripts and marked the passages that discussed the 
impact of the adverse event on the informant, the organisational support needed and the organisational support received. Other passages relevant to the overall aim of the study were also marked. These passages were then coded so as to capture the informants' ideas and the thematic concepts of the research. In discussions, the research group classified the coded passages into broader categories that were later divided into subcategories. To make sure that our categories represented the interview data faithfully, we compared our categories with the transcribed interview data. We used QSR NVivo software for data coding and categorisation.

In the Results section (see below), we use the following terminology to tally the number of informants' responses for a category or statement: 'few' for 1-4, 'some' for 5-9, 'half' for 10-11, 'most/the majority' for 12-20 and 'all' for 21. The informants are quoted in the Results section to give examples of how categories and subcategories were derived. Informants, both men and women, from different professions, with various years of practice and various adverse events are quoted. Based on the above sample, we selected quotations that reflect the thinking of many informants. All quotes were translated from Swedish to English.

The 21 adverse events were classified in accordance with the classification system used by the NBHW: drug treatment (eight events), diagnostics (four events), invasive procedures including operations (three events), suicide (one event) and other (unnecessary measures, no measures taken, delayed or insufficient measures) (five events).

The patient outcomes were classified as follows: death (six events), permanent injury (two events), short-term harm but no permanent injury (nine events), no harm to the patient (two events), no medical injury but the patient was offended (one event) and no information on the outcome for the patient (one event). Even when the patient was not harmed, the hospital had classified the event as a risk situation for the patient and consequently the event was reported.

\section{RESULTS}

This section begins with a presentation of how the informants described the impact of their involvement in an adverse event. Thereafter, we present their thoughts, as expressed in the interviews, on the organisational support they needed and the organisational support they received.

\section{The impact of the event}

With a few exceptions, the informants reported that the event had affected them on a personal and a professional level. The informants described similar reactions to these events even though the events differed, as did the intensity of their reactions. Although the interviews were conducted between 1 and 3 years after the events, all informants recalled the events in great detail.

Three subcategories related to the impact of the event were identified: (a) emotional reactions; (b) professional performance and self-confidence; and (c) duration of impact.

Emotional reactions

Some informants described initial reactions of shock and disbelief following the adverse event. They could not believe what had happened and that they had had a part in it. The majority of informants reported emotional reactions such as sadness, anxiety and reliving the event (flashbacks). Many said that they had mentally repeated the sequence of events over and over again:

I kept coming back to that day. (Interviewee No 4, Profession: Nurse, Type of adverse event: Too early discharge from hospital)

Other frequently reported reactions were guilt, shame and the feeling that one's professional reputation had been damaged:

I felt terribly ashamed that I had made a mistake. (Interviewee No 3, Profession: Nurse, Type of adverse event: Wrong medication dose)

Some informants worried about criticism from other people, while others were mainly self-critical. Feelings of frustration and sleep disturbances were other reactions.

A few informants reported serious health problems following the adverse event. They experienced clinical depression that required professional treatment and extended sick leave. At least two people still had not recovered fully at the time of the interview. The informants reported various factors that had influenced their health problems, such as feelings of guilt and shame, lack of support and private stressors. A few other informants also sought professional help although they continued to work full time.

Professional performance and self-confidence

Many informants felt insecure in their professional roles following the adverse event, especially immediately after the event, but for some this insecurity lasted longer. They said they doubted their professional judgment and sometimes even their career choice:

The security is gone in a way (...) you start to question yourself: Is it right what I'm doing? Is it time to change professions? (Interviewee No 4, Profession: Nurse, Type of adverse event: Too early discharge from hospital)

These reactions were not always linked to the patient outcome. Some informants were shattered by 
thought of what might have been. 'What if?' (the worst had happened) was a recurrent theme.

A majority of the informants reported that the adverse event affected their work to some extent. More than half described taking extra care in performing their work afterwards so as to avoid problems. While most informants reported they could continue their work much as they had before the event, one-third described how the emotional reactions made their work more difficult. Some informants expressed worries about disciplinary action or job loss.

\section{Duration of impact}

A majority of the informants described the overall impact of the adverse event as long-lasting. Their descriptions ranged from 'a few months' to ' 1 year or more'. More than half said the event still came back to them from time to time. For some, the interview brought back memories, thoughts and emotions. The majority described how the event had left an impression that will stay with them:

The event will always affect me. I will probably never be unaffected by it. (Interviewee No 13, Profession: Nurse, Type of adverse event: Wrong medication dose)

\section{Individual needs}

Based on our questions about the informants' needs generally and specifically about organisational support, we identified two post-adverse event subcategories: (a) the need to talk and receive emotional support; and (b) the need to understand and learn from the event.

The need to talk and receive emotional support

All informants expressed a need to talk in great detail about the adverse event:

Systematically go through: What has happened? Who did what? How and where and when? (Interviewee No 5, Profession: Doctor, Type of adverse event: Violation of confidentiality)

Only a few informants said they were given this opportunity in a structured way. What many needed the most was for other people to listen and to show empathy.

Even though most were supported by colleagues and family, the results highlight a need for support and understanding from their manager/employer. Many informants stressed the importance of follow-up by management. Some thought that ideally this follow-up should take place more than once because their needs may differ short-term and long-term:

I can think of it as (...) somewhat similar to when people are mourning. Then it is good to follow-up, and not expect everything to be normal after for a while. (Interviewee No 7, Profession: Doctor, Type of adverse event: Wrong medication dose)

The need to understand and learn from the event

Besides the need to receive emotional support, many expressed a need to understand and learn from the event:

There are two parts: one is the question of knowledge. Is there any knowledge that we don't have and that could have been useful in this case? And the other is how you take it as a person - the discomfort so to speak. (Interviewee No 9, Profession: Doctor, Type of adverse event: Operation went wrong)

Many informants stated that understanding is a prerequisite for learning from past events, on both an individual and an organisational level. Some described the need to talk about work conditions that they thought, directly or indirectly, caused the event. Two such conditions mentioned were understaffing and poor routines. These informants were particularly frustrated that the investigation did not really consider the root causes of the adverse event.

For some informants, it was especially important that they received professional reassurance and that their actions and decisions were confirmed:

I think you need to hear that you are a good doctor. I think you need that kind of reassurance. (Interviewee No 18, Profession: Doctor, Type of adverse event: Operation went wrong)

\section{Organisational support}

The majority of the informants expressed a need for support after the adverse event. Three subcategories related to organisational support were identified: the importance and extent of (a) management/institutional support, (b) peer support, and (c) the investigation process.

\section{Management/institutional support}

Five of the 21 informants reported they received the support they needed from the hospital management. The majority, however, reported insufficient or no support from their closest manager or any hospital representative. Most informants turned to their colleagues and/or family for support, while some were reluctant to disclose to others what had happened, leaving them isolated with their feelings. Some were disappointed in their employer, but most pointed to the lack of structures and routines for handling these events and for supporting staff:

Well, there are no guidelines. From my perspective, there is no structure; this is how we do it. It is very personal. And if you are lucky, you meet the right people. And if you are unlucky, you meet the wrong people. (Interviewee No 13, Profession: Nurse, Type of adverse event: Wrong medication dose) 
The majority described a lack of open discussions about adverse events in the workplace. Those who reported a more positive view of organisational support usually described the personal characteristics of their manager or of their specific department. Some informants had been referred for professional help, while others stated that such support should be more visible. Even when support was available, some expressed difficulties accepting support:

I think there is an inner resistance towards getting external help. At least, among doctors [the idea is] "I can handle this" (...) but I think that really we should have much more general support. In difficult situations overall. Not only after adverse events. (Interviewee No 18, Profession: Doctor, Type of adverse event: Operation went wrong)

\section{Peer support}

A majority of the informants described peer support as crucial after the adverse event. Most colleagues had been empathic and understood that the event could have happened to them. Sharing with non-judgmental colleagues was reported to ease the emotional burden:

I really want to highlight how important that support is (...) without it, I don't know where I would have been now (...), if I would have ever dared to come back and work as a nurse again. (Interviewee No 14, Profession: Nurse, Type of adverse event: Wrong medication dose)

In those cases where peer support failed, the informant found it more difficult to cope with the event. About one-third reported that the event had had a negative impact on their relationships with colleagues. Some had experienced tension and even open conflict with critical and judgmental comments from others.

\section{The investigation process}

Although our interview did not address the formal process of reporting and investigating the adverse event, many informants raised the investigation process and its inadequacies. Poor routines in this process had added to the informants' emotional distress in several cases. Most informants were unclear on the procedures for reporting to the NBHW. The majority did not receive enough information on the steps of this process.

Many informants reported a lack of follow-up after the conclusion of the investigation. The investigations could take several months or even more than a year. Even when the NBHW had reached a decision and had closed the case, half of the informants said they had not been notified. This left many questions unanswered:

What does this mean for me? What happened to the patient? With the other people involved? How serious is this? (Interviewee No 2, Profession: Nurse, Type of adverse event: Wrong medication dose)

Some informants worried about disciplinary action or job loss. The long investigation time and lack of follow-up increased the difficulty in recovering from the adverse event and reaching closure.

\section{DISCUSSION}

This study was designed to investigate how healthcare professionals at a Swedish university hospital were affected by their involvement in adverse events, with particular emphasis on their need for organisational support and how well the organisation meets those needs.

One major finding to emerge from this study was that most informants experienced emotional distress following the adverse events, in a way that confirms previous research. ${ }^{1}{ }^{2}{ }^{8-14}$ The majority described the overall impact as long-lasting and referred to the event as a memory that will stay with them.

The second major finding was that the extent of the impact on the healthcare professional was related to the organisation's response to the event. Inadequate support and a lack of a clear investigation seemed to deepen and prolong the impact. Consistent with previous research, ${ }^{10-12}$ our findings confirm that patients and professionals may be affected in two ways after an adverse event: first, by the incident itself, and second, by the manner in which the incident is handled.

Related to the above, the majority of the informants reported inadequate organisational support and, when given, the support was often unstructured and unsystematic. A clear lack of employer follow-up on the needs of the staff member and on the formal investigation process was revealed. These combined findings provide circumstantial evidence that this dual lack of follow-up made it more difficult to emotionally process the event and reach closure.

The third major finding was the specific needs the informants had after the adverse event. The most critical need was to talk with others about what had happened. In such conversations the informants could share the emotional burden and receive personal and professional reassurance. There was also a strong need to understand the event and learn from it, often through discussions with others. Other research has identified similar needs. ${ }^{15} 7{ }^{11}$ When these needs are not met, individuals may be left isolated with their feelings and suffer in silence. Failure to meet those needs may also hinder opportunities to learn from these events on both an individual and an organisational level.

A final finding was that open disclosure about adverse events was not routine within organisations and shared between staff members. Previous research shows similar results. For example, Wu et al report that even when adverse events were discussed in a 
work group, "the tough issues were not addressed" $(\mathrm{p} 2093)^{5}$. Previous research has discussed the perfectionism expected of healthcare professionals. 7101623 Consequently, staff may be reluctant to speak openly and seek help after an adverse event for fear of being stigmatised. ${ }^{1} 1112$ Therefore, given the potential harm to second victims when they shut themselves off from support, mandatory routines are necessary following adverse events.

Our collection of findings implies the need for adequate support systems that help staff and provide learning opportunities for the organisation. By simultaneously paying attention to both the handling of individual needs and the formal investigation, such systems will likely contribute to the positive development of an organisational culture of patient safety. Future work needs to be done to further explore the practical requirements and design of such a system. In Sweden, healthcare organisations have recently started measuring patient safety culture by using a version of the Hospital Survey on Patient Safety Culture questionnaire. ${ }^{24}$ The Swedish version includes two new dimensions: 'Information and support to patients and family who have suffered an adverse event' and 'Information and support to staff who have been involved in an adverse event'. Both questions address the relationship between the patient safety culture and how the first and second victims of adverse events are taken care of. ${ }^{25}$ This information can be used to develop support structures which can help counter unnecessary distress for many professionals involved in adverse events. The use of stable and transparent procedures for the investigation and analysis of adverse events seems to be a central feature of any support system. Based on our findings we believe that such procedures, together with timely and continuous emotional support from managers and colleagues, provide adequate support for most professionals involved in these events. In some cases, however, more extensive psychological help may be needed.

\section{LIMITATIONS}

We collected data at one hospital in Sweden from 21 healthcare professionals. The relatively small number of informants makes our findings mostly relevant to this setting. As shown, a case study like ours can still provide in-depth understanding of a particular system or phenomenon, and pinpoint specific contextual factors that need to be considered when applying the insights elsewhere. Another limitation relates to the fact that because the informants were volunteers, there is an issue of self-selection bias. However, despite the relatively small sample size, our informants varied in profession, gender, years in practice and also regarding the nature and outcome of the adverse event. Nevertheless, general patterns could be identified. Future research should be designed to include larger samples of professionals involved in an adverse event to further explore the magnitude and nature of the current problems.

\section{CONCLUSIONS}

We stress the need to further recognise the nature of the second victim phenomenon and the need for organisational support for affected healthcare professionals. We conclude that attention should be paid to the organisational climate where these issues should be addressed and discussed in a non-judgmental manner. Our findings underline the need for wellestablished support structures that can meet the needs of involved individuals. The support structures have to include both timely and transparent procedures for the investigation and analysis of adverse events, and areas where staff involved in adverse events can meet with colleagues to share their emotions and receive personal and professional reassurance. These issues are critical for all hospital managers and policy makers who wish to prevent and manage adverse events and to promote a positive safety culture.

Contributors SU, MAS, JØ: conception and design of the study, data collection and analysis; JH, MB: data analysis; SU, MAS, $\mathrm{JH}$ : writing the article; JØ, MB: article revision.

Funding Vetenskapsrådet/The Swedish Research Council provided funding for this study.

Competing interests None.

Ethics approval The Regional Ethical Review Board of Stockholm and the healthcare employees' union at the hospital approved this study.

Provenance and peer review Not commissioned; externally peer reviewed.

Open Access This is an Open Access article distributed in accordance with the Creative Commons Attribution Non Commercial (CC BY-NC 3.0) license, which permits others to distribute, remix, adapt, build upon this work noncommercially, and license their derivative works on different terms, provided the original work is properly cited and the use is non-commercial. See: http://creativecommons.org/licenses/by$\mathrm{nc} / 3.0$ /

\section{REFERENCES}

1 Schwappach DLB, Boluarte TA. The emotional impact of medical error involvement on physicians: a call for leadership and organisational accountability. Swiss Med Wkly 2009;139:9-15.

2 Seys D, Scott S, Wu A, et al. Supporting involved health care professionals (second victims) following an adverse health event: a literature review. Int J Nurs Stud 2013;50:678-87.

3 Zegers M, de Bruijne MC, Wagner C, et al. Adverse events and potentially preventable deaths in Dutch hospitals: results of a retrospective patient record review study. Qual Saf Health Care 2009;18:297-302.

4 Soop M, Fryksmark U, Köster M, et al. The incidence of adverse events in Swedish hospitals: a retrospective medical record review study. Int J Qual Health Care 2009;21:285-91.

$5 \mathrm{Wu}$ AW, Folkman S, McPhee SJ, et al. Do house officers learn from their mistakes? JAMA 1991;265:2089-94.

6 Christensen JF, Levinson W, Dunn P. The heart of darkness. The impact of perceived mistakes on physicians. J Gen Intern Med 1992;7:424-31. 
7 Newman MC. The emotional impact of mistakes on family physicians. Arch Fam Med 1996;5:71-5.

8 Wolf ZR, Serembus JF, Smetzer J, et al. Responses and concerns of healthcare providers to medication errors. Clin Nurse Spec 2002;14:278-90.

9 Rassin M, Kanti T, Silner D. Chronology of medication errors by nurses: accumulation of stresses and PTSD symptoms. Issues Ment Health Nurs 2005;2:1-4.

10 Waterman AD, Garbutt J, Hazel E, et al. The emotional impact of medical errors on practicing physicians in the United States and Canada. Jt Comm J Qual Saf 2007;22:467-76.

11 Scott SD, Hirschinger LE, McCoig M, et al. The natural history of recovery for the healthcare provider "second victim" after adverse patient events. Qual Saf Health Care 2009;18:325-30.

12 Edrees HH, Paine LA, Feroli ER, et al. Health care workers as second victims of medical errors. Pol Arch Med Wewn 2011;121:101-8.

13 Gazoni F, Amato PE, Malik ZM, et al. The impact of perioperative catastrophes on anesthesiologists: results of a national survey. Anesth Analg 2012;113:596-603.

14 Stangierski A, Warmuz-Stangierska I, Ruchata M, et al. Medical errors-not only patients' problem. Arch Med Sci 2012;8:569-74.

15 Aasland OG, Forde R. Impact of feeling responsible for adverse events on doctors' personal and professional lives: the importance of being open to criticism from colleagues. Qual Saf Health Care 2005;14:13-17.
16 Wu AW. Medical error: the second victim. The doctor who makes a mistake needs help too. BMJ 2000;320:726-27.

17 West CP, Huschka MM, Novotny PJ, et al. Association of perceived medical errors with resident distress and empathy: a prospective longitudinal study. JAMA 2006;296:1071-78.

18 Shanafelt TD, Balch CM, Dyrbye L, et al. Special report: suicidal ideation among American surgeons. Arch Surg 2011;146:54-62.

19 Aleccia J. Nursés suicide highlights twin tragedies of medical errors. msnbc.com web site. http://www.msnbc.msn.com/id/ 43529641/ns/health-health_care/ (accessed 27 Jun 2011).

20 Arndt M. Medication errors. Research in practice: how drug mistakes affect self-esteem. Nurs Times 1994;90:27-30.

21 Graneheim UH, Lundman B. Qualitative content analysis in nursing research: concepts, procedures and measures to achieve trustworthiness. Nurse Educ Today 2004;24:105-12.

22 Shannon SE, Hsieh HF. Three approaches to qualitative content analysis. Qual Health Res 2005;15:1277-88.

23 Paparella S. Caring for the caregiver: moving beyond the finger pointing after an adverse event. J Emerg Nurs 2011;37:263-65.

24 Nieva VF, Sorra JS. Safety culture assessment; a tool for improving patient safety in healthcare organizations. Qual Saf Health Care 2003;12(Suppl 2):ii17-23.

25 Hedsköld M, Pukk-Härenstam K, Berg E, et al. Psychometric properties of the hospital survey on patient safety culture, HSOPSC, applied on a large Swedish health care sample. BMC Health Serv Res 2013;13:332. 